NASA/TM-1999-208842

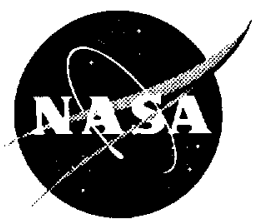

\title{
Preliminary Results of Solid Gas Generator Micropropulsion
}

Wilhelmus A. de Groot

Dynacs, Inc., Brook Park, Ohio

Brian D. Reed

Glenn Research Center, Cleveland, Ohio

Marshall Brenizer

Massachusetts Institute of Technology, Cambridge, Massachusetts

Prepared for the 34th Joint Propulsion Conference cosponsored by the AIAA, ASME, SAE, and ASEE Cleveland, Ohio, July 13-15, 1998

National Aeronautics and Space Administration

Glenn Research Center 
This report is a formal draft or working paper, intended to solicit comments and ideas from a technical peer group.

Trade names or manufacturers' names are used in this report for identification only. This usage does not constitute an official endorsement, either expressed or implied, by the National Aeronautics and Space Administration.

Available from

NASA Center for Aerospace Information

7121 Standard Drive

Hanover, MD 21076

Price Code: A03
National Technical Information Service 5285 Port Royal Road Springfield, VA 22100 Price Code: A03 


\title{
Preliminary Results of Solid Gas Generator Micropropulsion
}

\author{
Wim A. de Groot* \\ Dynacs Engineering Company, Inc. \\ Brook Park, Ohio \\ Brian D. Reed \\ National Aeronautics and Space Administration \\ Glenn Research Center \\ Cleveland, Ohio \\ Marshall Brenizer \\ Massachusetts Institute of Technology \\ Cambridge, Massachusetts
}

\begin{abstract}
A decomposing solid thruster concept, which creates a more benign thermal and chemical environment than solid propellant combustion, while maintaining performance similar to solid combustion, is described. A Micro-ElectroMechanical thruster concept with diode laser and fiber-optic initiation is proposed, and thruster components fabricated with MEMS technology are presented. A high nitrogen content solid gas generator compound is evaluated and tested in a conventional axisymmetric thrust chamber with nozzle throat area ratio of 100 . Results show incomplete decomposition of this compound in both low pressure $(1 \mathrm{kPa})$ and high pressure (1 $\mathrm{MPa}$ ) environments, with decomposition of up to $80 \%$ of the original mass. Chamber pressures of $1.1 \mathrm{MPa}$ were obtained, with maximum calculated thrust of approximately $2.7 \mathrm{~N}$. Resistively heated wires and resistively heated walls were used to initiate decomposition. Initiation tests using available lasers were unsuccessful, but infrared spectra of the compound show that the laser initiation tests used inappropriate wavelengths for optimal propellant absorption. Optimal wavelengths for laser ignition were identified. Data presented are from tests currently in progress. Alternative solid gas generator compounds are being evaluated for future tests.
\end{abstract}

\footnotetext{
${ }^{*}$ Sr. Research Engineer, Senior Member AIAA

${ }^{+}$Research Engineer. Member AIAA

$\stackrel{\ddagger}{\ddagger}$ Student
}

\section{Introduction}

One approach in the continuous quest to reduce spacecraft launch costs is the miniaturization of spacecraft and spacecraft subsystems. The development of microsized subsystems and components that operate without a reduction in performance relative to their full sized counterparts requires a substantial investment and an abundance of innovative engineering, aided by some breakthrough developments in materials science and manufacturing technologies.

The need for a substantial investment and for innovative engineering is especially true for the development of a micro-spacecraft propulsion subsystem. Propulsion systems of most operational spacecraft occupy a significant mass and volume fraction (from below $50 \%$ to over $90 \%$ ), most of which is consumable propellants. Miniaturizing the propellant storage and feed system causes the percentage dry weight to increase, simply because the surface to volume ratio increases, and also because microcomponents are proportionally heavier then their larger counterparts.

In addition to the dry weight penalty, micropropulsion systems generally suffer a degradation in performance, as expressed by a reduced specific impulse. A larger surface to volume ratio 
increases the heat transfer at the walls. Wall material thermal limitations and incomplete propellant mixing (bipropellants) cause the performance to decrease. The performance degradation varies depending on the propulsion concept. Therefore, micro-sizing is a useful concept for only a tew propulsion concepts.

Micro-propulsion is currently an active field of research. Among the propulsion systems under investigation for miniaturization are cold gas thrusters, ${ }^{1}$ subliming solids thrusters, ${ }^{2}$ resistojets, ${ }^{2}$ ion thrusters, ${ }^{3}$ bi-propellant, ${ }^{4}$ and solid propellant thrusters. ${ }^{5}$ Cold gas thrusters and subliming solid thrusters are simple and inexpensive. The major drawbacks of cold gas and subliming solid thrusters are the low specific impulse (Isp). Cold gas thrusters have the added disadvantage that the high leak rate imposes short mission durations. Miniature resistojets have higher Isp than both cold gas and subliming solid concepts but are more complex and require power and some power conditioning. Micro-ion thruster concepts are complex, require a substantial power (relative to size), and present a challenge from the perspective of insulation, efficiency, and power conditioning. Bi-propellant thrusters are in an early stage of investigation but are complex and present substantial technical challenges. Micro-solid propellant thrusters are simple, inexpensive, and have an acceptable performance but can not be throttled or actively controlled.

The focus of propulsion system miniaturization has shifted to micro-electromechanical systems (MEMS) technology for manufacturing. Among the advantages of this manufacturing technology are the low cost of batch processed items and the ability to scale down to micrometer size features. The use of this manufacturing technology currently limits the materials to silicon and silicon carbide. Material properties limit the propulsion concept feasibility. Most concepts studied create harsh environments in which all material properties are tested to their extremes and heyond.

The goal of the current program is to evaluate whether MEMS technologies could be used to produce low cost, compact, simple, high performance propulsion systems appropriate to deliver impulse requirements for micro-satellites. An evaluation of chemical propulsion concepts ${ }^{5}$ indicated that the use of a gas generator solid appeared to provide a balance between the desired high performance. high thrust, and a relatively benign environment, with a promise of low complexity and low cost. The major drawback is the lack of restartability and the twodimensional nature of MEMS manufactured parts which could reduce performance.

This paper presents an introduction to the concept, propellant properties, ignition candidates, MEMS designs, test articles, and some preliminary results.

\section{Solid Gas Generator Propulsion Concept}

Decomposing solid propellants offer several advantages over combusting solid propellants for micro-rocket applications. First, the decomposing solids do not suffer from the possibly violent instability associated with many combusting solid propellant grains. Due to the high temperature and rapid burning rate of combusting solids, the local pressure on the burning surface can oscillate by as much as $30 \%$ of the chamber pressure. The localized high pressure in cracks and grooves in the surface can cause the temperature and burning rate to drastically increase, undermining the stability of the propellant as cracks and fissures propagate. This instability increases the heat flux to the surface of the propellant, the nozzle walls and the propellant storage case. ${ }^{6}$

Due to the small size and materials choice for micro-rockets, this high chamber temperature and high heat flux associated with combustion can cause severe structural problems. Decomposing solids, which react at a lower temperature and slower burning rate, can provide adequate performance while maintaining the integrity of the micro-rocket structure. ${ }^{7}$ The slower burning rate allows most of the thermal energy released in the decomposition process to be converted directly into the kinetic energy of the gaseous products. ${ }^{8}$ The heat is then convected out of the nozzle with the exhaust gas, resulting in lower heat transfer to the walls of the microrocket itself. There is no energy released in the form of light from a flame, and the highest local temperature is on the surface of the solid itself, rather than in the gases within the combustion 
chamber. Therefore, the decomposition process maintains an overall lower combustion chamber temperature.

Additionally, the decomposition process is irreversible, unlike the sublimation of other gas generator processes. In sublimation, as the temperature decreases in the nozzle, the solid can reform, clogging the thruster. Decomposing gas generators theoretically decompose fully into gaseous products that will not re-solidify or produce smoke as in combustion. Decomposing solids offer a more stable, less complex, less toxic alternative for use as propellants in microthrusters.

A cross sectional drawing of a MEMS based solid gas generator thruster is shown in Figure 1. The structural material is silicon. Chamber dimensions are $6.35 \mathrm{~mm}$ diameter with $550 \mu \mathrm{m}$ height. The contraction angle is $49.5^{\circ}$. The throat dimensions are $300 \mu \mathrm{m}$ wide by $550 \mu \mathrm{m}$ high by $300 \mu \mathrm{m}$ long. The nozzle expansion angle is $27.7^{\circ}$. Because it is not known at these Reynold's numbers $\left(\operatorname{Re} \sim 10^{4}\right)$ whether the thrust to weight ratio warrants extended nozzles, several nozzle expansion ratios were manufactured. Expansion ratios of $1,5,10,15,20$, and 25 are available. Different size filters upstream of the nozzle are designed to prevent particulate matter from blocking the throat, which would lead to catastrophic failure.

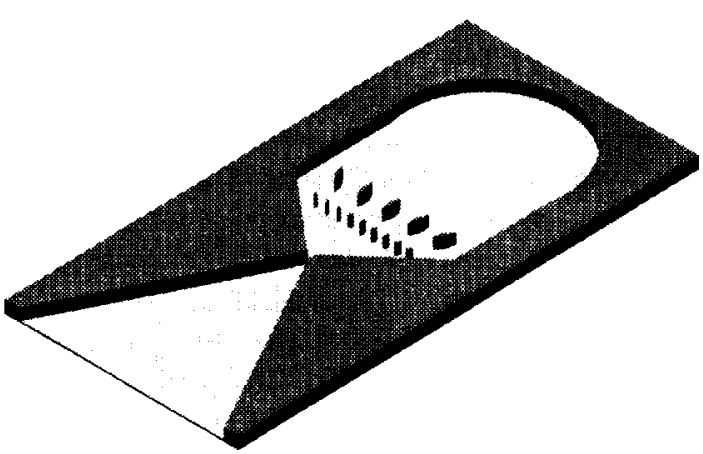

Figure 1: Micro-ElectroMechanical
Thruster Design.

A gas generator pellet resides inside the chamber. Decomposition is started with a fiber pigtailed diode laser. Silicon is transparent to infrared radiation at wavelengths above $1.3 \mu \mathrm{m}$. A $8 \mu \mathrm{m}$ diameter optical fiber transmits the laser beam from the laser to the thruster. A graded index lens, mounted at the fiber exit, refocuses the light through the thruster wall onto the compound. This arrangement leaves the wall structurally sound. No holes for wires or components are required. Multiple thrusters can be ignited by the same diode laser, by switching the laser beam to different fibers.

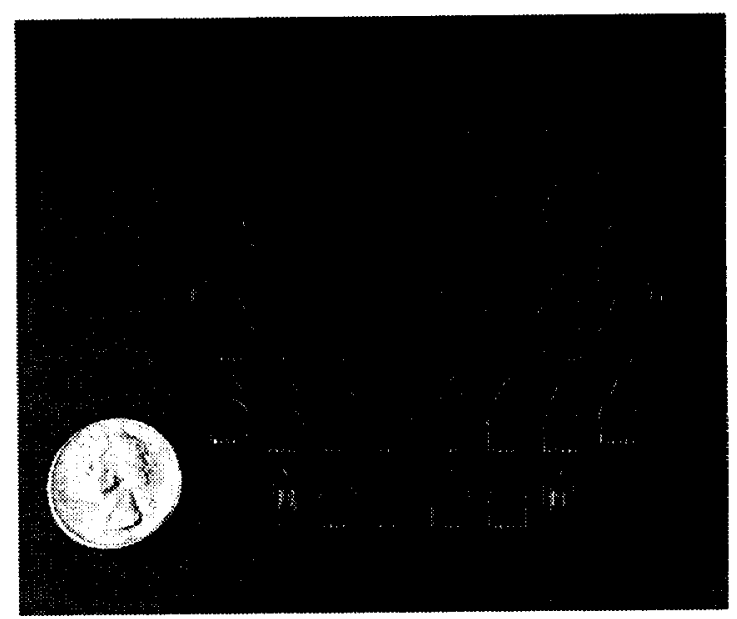

Figure 2: Batch Fabricated MEMS Micro-Thrusters.

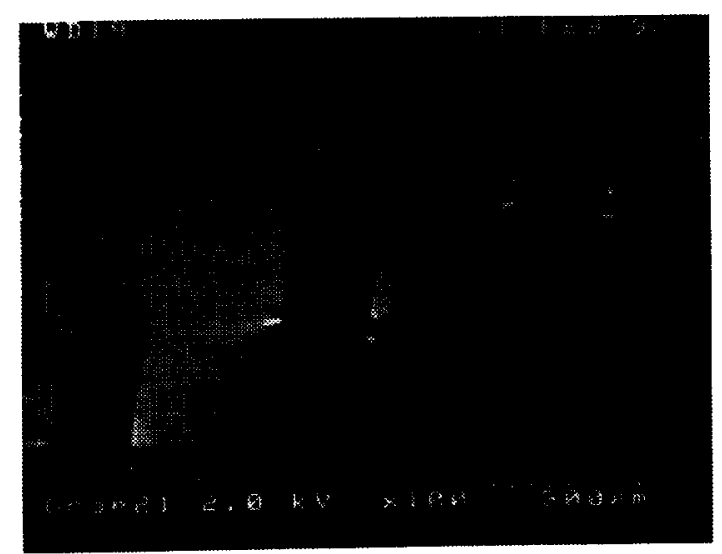

Figure 3: Detail of Comb Filter of MEMS Micro-Thruster.

Thrusters can be batch fabricated, lowering the cost per thruster. An example of batch fabricated thrusters, with varying nozzle area ratio, is shown in Figure 2. A $125 \mathrm{~mm}$ diameter, $500 \mu \mathrm{m}$ thick silicon wafer is etched to contain 36 half thrusters, or 18 thrusters. Scribe lines etched in 
the wafer allows easy separation of the thruster halves. Depth of etching is $300 \mu \mathrm{m}$. Deeper etching undermines the structural integrity of smaller details, such as the filter elements shown in Figure 3.

\section{Test Facility}

All tests described in this paper were performed inside a test vessel, schematically shown in Figure 4. Pressure inside the vessel could be varied from $5 \times 10^{3}$ to $5 \times 10^{6} \mathrm{~Pa}$, with a nitrogen inert atmosphere. A small sample vessel was used to extract gas products for analysis. A high frequency pressure transducer was used to monitor pressure changes inside the vessel and during subsequent tests inside the decomposition chamber described later. An additional pressure transducer monitored pressure inside the vessel.

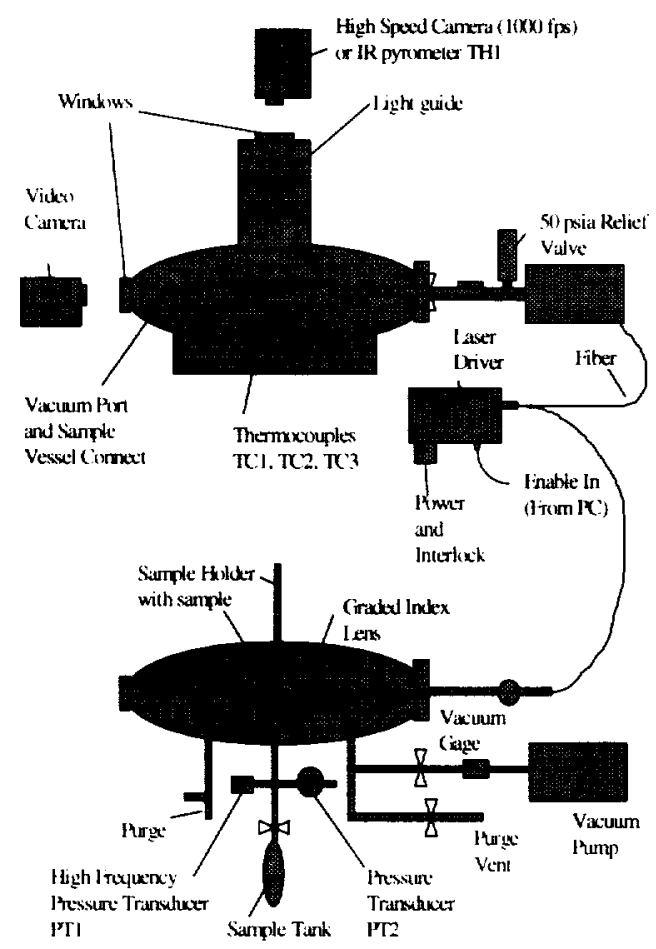

Figure 4: Test Vessel.

Several thermocouples monitored temperatures at different locations inside the test facility. The slow response of the thermocouples, as compared to the decomposition, caused the temperatures recorded to be only of value for the determination of the temperature at which decomposition started. A video camera was used to record decomposition tests. A high speed camera monitoring port is provided but has not been utilized.

Tests performed to evaluate gas generator compound initiation temperature and gaseous product composition were conducted by mounting one or more pellets on a nichrome wire holder. The pellets were held in place with a "diamond setting", four wires bending towards the pellet. The nichrome wire was resistively heated, with wire temperature continuously monitored with a thermocouple. Decomposition temperatures and gas samples were obtained for each of the tests.

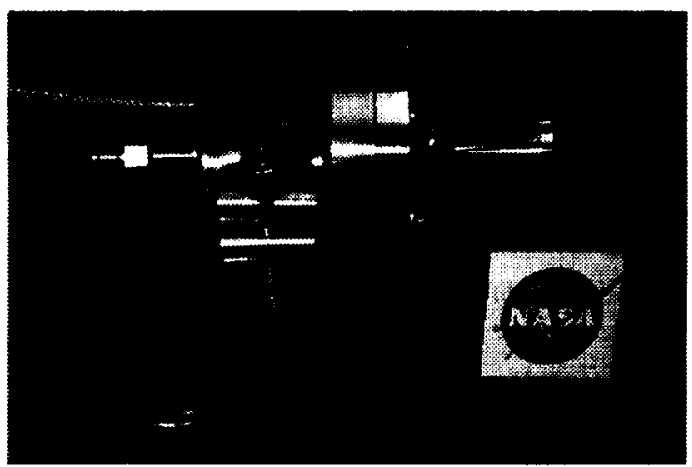

Figure 5: Axisymmetric Miniature Thruster.

Alternate tests were performed with pellets mounted inside available thruster hardware. A small, conventionally machined axisymmetric thruster with trumpet nozzle of area ratio of 100 , was used during a previous program to study low Reynolds numbers nozzle flows. ${ }^{9}$ This thruster, shown in Figure 5, was modified with a copper chamber insert that had the purpose of accommodating one or several pellets, as well as functioning as a heating element. A high frequency pressure transducer and thermocouple were installed to monitor chamber conditions. Screens between the pellets and the throat were installed to prevent throat blockage by larger particles. The thrust chamber diameter was approximately $6.5 \mathrm{~mm}$ with a length of $25 \mathrm{~mm}$. The throat diameter was $1.51 \mathrm{~mm}$. The thruster was installed inside the test facility where ambient conditions could be controlled. 


\section{Propellant}

The solid gas generator compound used is a heterocyclic nitrogen compound with chemical formula 3,6-Diamino-1,2,4,5-tetrazine 1,4dioxide $\left(\mathrm{C}_{2} \mathrm{H}_{4} \mathrm{~N}_{6} \mathrm{O}_{2}\right)$ and trade name LAX $112 .{ }^{11}$ The structure of this reddish brown powder is shown in Figure 6. The compound is synthesized in $40 \mu \mathrm{m}$ size particles. Pellets of approximately $100 \mathrm{mg}$ are created in a press. The compound's heat of formation is $164 \mathrm{~kJ} /$ mole and the specific weight is $1.86 \mathrm{gm} / \mathrm{cc}$. The compound decomposes into nitrogen, hydrogen and carbon monoxide with an average molecular weight of $20.8 \mathrm{~g} / \mathrm{mole}$. Surface temperature at decomposition is $2235 \mathrm{~K}$. The heat released at the surface sustains the decomposition, the rate of which increases with increasing pressure.

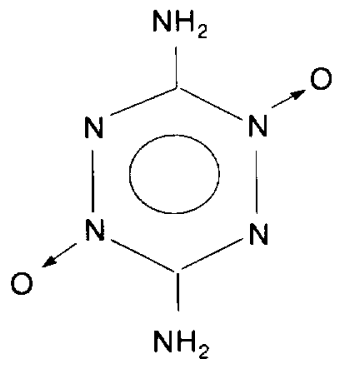

Figure 6: 3,6-Diamino-1,2,4,5-tetrazine 1,4-dioxide Scheme.

The compound is electrostatic and impact insensitive and is not affected by storage in a humid or carbon dioxide rich environment. Furthermore, it is not toxic or carcinogenic. The decomposition process can be initiated by an electrically heated wire, or a focused laser beam. Differential thermal analysis shows that decomposition begins at $220^{\circ} \mathrm{C}{ }^{10}$

Assuming a nozzle area ratio of 100 , the theoretical performance of the compound as rocket propellant is between $262 \mathrm{sec}$ Isp for equilibrium flow and $244 \mathrm{sec}$ Isp for frozen flow. Initial decomposition tests performed to evaluate the compound for air bag use showed that the decomposition products approach the frozen flow gas mixture (partial measurement showed $46.5 \%$ nitrogen, $29 \%$ carbon monoxide, and $0.5 \%$ oxygen by weight).

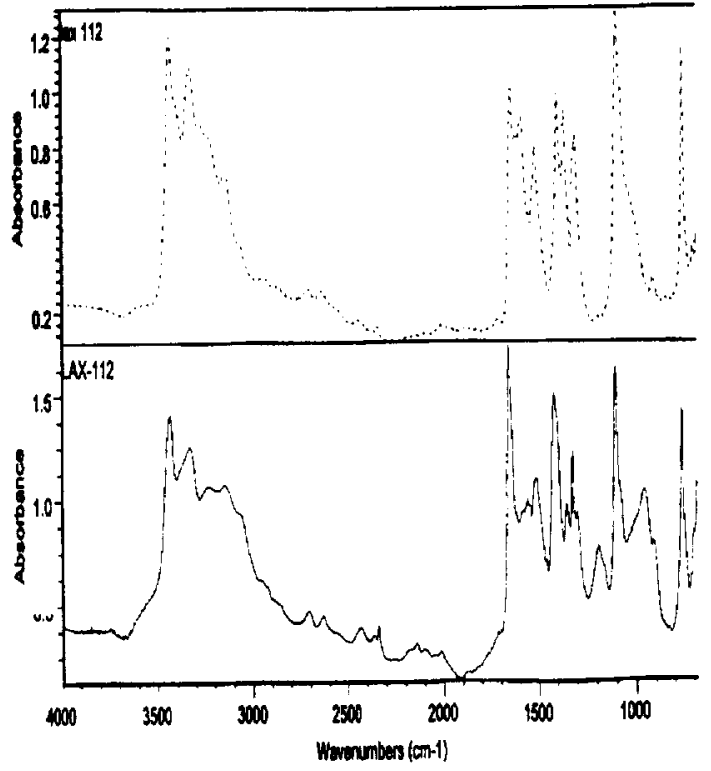

Figure 7: Gas Generator Compound
FT-IR Spectra.

Samples of the compound were collected and analyzed with a FT-IR microscope. Two characteristic spectra are shown in Figure 7. It is surprising that, even though most samples showed identical spectra, some samples differed, suggesting that the compound was not pure. This could provide some of the answers to questions raised by the test results. The two spectra show some of the differences found, even though these differences are not discussed here. The fundamental infrared frequencies given in lirterature $^{10}$ are clearly visible. They are at $v$ values of $3407,3288,3139,1623,1513,1371$. 1311,1057 . and $748 \mathrm{~cm}^{-1}$. These values also indicate the wavelengths of maximum absorption, which are helpful to determine strategies for laser ignition of the compound.

\section{Experimental Results}

Ten tests were completed for which the initiation temperature was succesfully recorded. Five tests used nichrome wire heating and five tests used copper plate heating. The temperature at which initiation of the decomposition occurred was a constant $188{ }^{\circ} \mathrm{C}$ using nichrome wire heating. whereas the copper block heating varied from $165^{\circ} \mathrm{C}$ to $192^{\circ} \mathrm{C}$. This variation is considered to be of secondary importance if an ignition method 
can be found which is instantaneous, independent of the temperature.

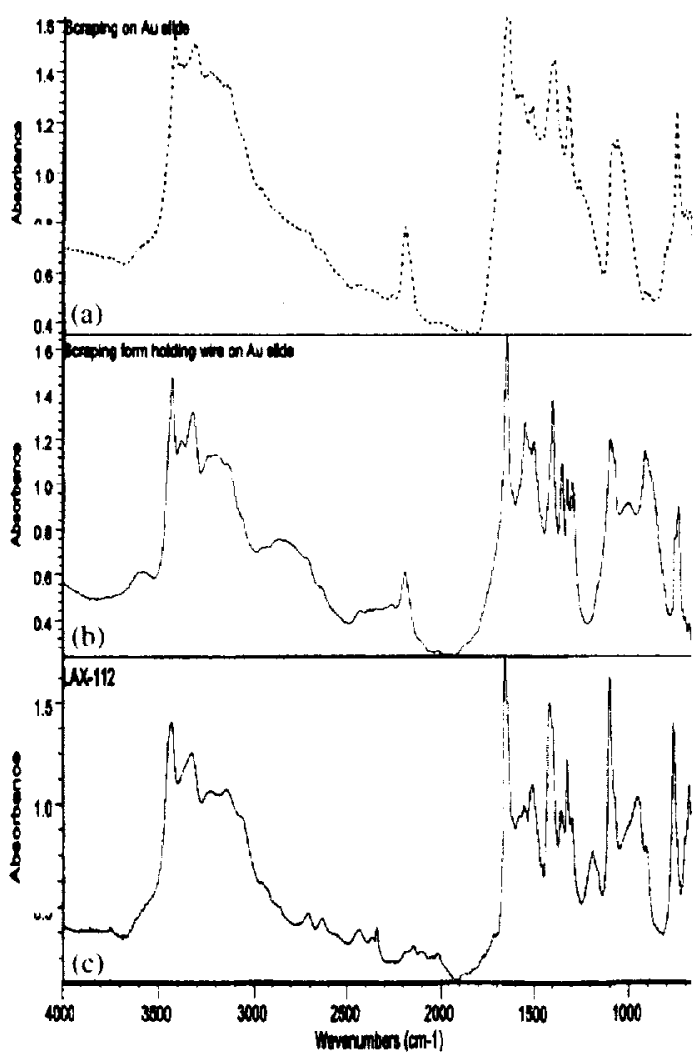

Figure 8: FT-IR Spectra of Incompletely Decomposed Gas Generator Compound Collected at Different Locations: a. $100 \mathrm{~mm}$ from sample; b. $5 \mathrm{~mm}$ from sample; c. sample.

The tests using nichrome wire heating were performed inside the test facility, with $5 \mathrm{kPa}$ facility pressure. Video footage showed only partial decomposition, with large amounts of powder obscuring the video frames. Test samples of the gas products were collected and partially analyzed with a gas chromatograph. This analysis showed hydrogen, nitrogen, and oxygen, but did not show carbon monoxide.

Several samples of the powder which settled inside the chamber were taken after test completion and analyzed with an FT-IR microscope. Samples were taken at increasing distance from the sample mounting location.
Three spectra are shown in Figure 8. All three spectra display some of the dominant peaks of the original gas generator compound spectra. Also, a peak exists at approximately $2200 \mathrm{~cm}^{-1}$ in each of these spectra, which does not appear in the reference spectrum.

None of the powder samples collected exactly resembled the original compound. This, together with the hydrogen found in the gas products, suggests that partial decomposition occurred. Samples collected closer to the mounting location resemble the original spectra closer. This suggests less decomposition. It could be possible that heat was extracted from the decomposing powder by the surfaces that the powder settled on. This could have limited the decomposition. However, the likely cause of incomplete decomposition is the low pressure, which allows the heat of decomposition to be convected by the gas produced instead of conducted into the solid for continued decomposition.

In order to identify the low pressure as the sole culprit of incomplete decomposition, tests were performed with compound material mounted inside the combustion chamber of a miniature thruster. An initial test was performed with 0.1 MPa facility pressure and a single pellet. Three subsequent tests were performed with two pellets and $0.01 \mathrm{MPa}$ facility pressure. A generic thruster chamber pressure curve, obtained for each of these tests, is shown in Figure 9. A summary of the test results for the tests performed is shown in Table 1 . The peak pressure is reached in several tens of milliseconds, with a slow chamber pressure decay which lasts several hundreds of milliseconds.

Assuming that the chamber temperature at maximum pressure is approximately $1000 \mathrm{~K}$, and correcting for the overexpansion with the facility back pressure, then the thrust level at peak pressure for test \#2 is approximately $2.0 \mathrm{~N}$ and for test \# 5 is approximately $2.7 \mathrm{~N}$. Not enough data was available for the other tests to complete similar calculations. Calculations show that the mass decomposed is approximately 70 and 167 mg respectively, or $57 \%$ and $72 \%$ of the original mass. 
Preliminary laser ignition tests of LAX112 were not successful. One test involved exposure of the pellet surface to a $8 \mu \mathrm{m}$ diameter pulsed laser beam of $1 \mathrm{~mW}$ average laser power at $1.32 \mu \mathrm{m}$ wavelength. This represents a power density of 2 $\mathrm{kW} / \mathrm{cm}^{2}$. This falls within values reported in the literature for successful ignition. ${ }^{11.12}$ These values however were for a larger surface exposure, where heat could not he conducted through the surface.

An $87 \mathrm{~mW}$ Nd:Yag, lasing at $0.532 \mu \mathrm{m}$, was also used to test ignition. With a common planoconvex lens, the beam was focused on the pellet surface, covering an area with a diameter of 500 $\mu \mathrm{m}$, creating an average power density over the exposed spot of approximately $50 \mathrm{~W} / \mathrm{cm}^{2}$. After an exposure time of several minutes, the compound surface showed a dark red discoloration but did not decompose.

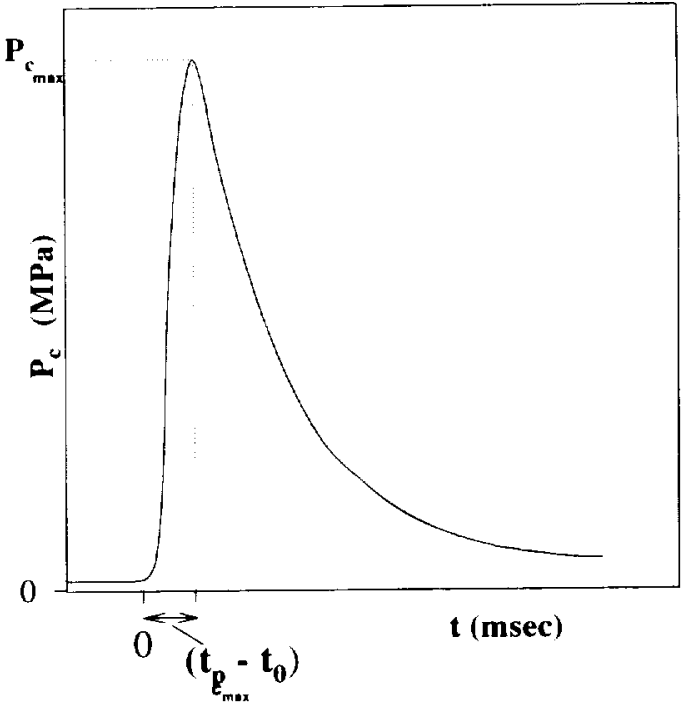

Figure 9: Chamber Pressure as a Function of Time

\begin{tabular}{|l|c|c|c|c|c|c|}
\hline Test \# & 1 & 2 & 3 & 4 & 5 & 6 \\
\hline \# Pellets & 1 & 1 & 1 & 2 & 2 & 2 \\
\hline Weight (mg) & 105.4 & 124.0 & 117.4 & 218.5 & 233.3 & 219.9 \\
\hline$P_{\text {facility }}(\mathrm{MPa})$ & 0.1 & 0.1 & 0.1 & 0.01 & 0.01 & 0.01 \\
\hline Peak $\mathrm{P}_{\mathrm{c}}(\mathrm{MPa})$ & 0.85 & 0.92 & 0.22 & 0.34 & 1.10 & 0.51 \\
\hline $\mathrm{T}_{\text {ini }}{ }^{\left({ }^{\circ} \mathrm{C}\right)}$ & 177 & 192 & 190 & 165 & 183 & 179 \\
\hline $\mathrm{t}_{\text {peak }} \mathrm{t}_{\mathrm{t}}(\mathrm{ms})$ & 18 & 18 & 63 & 55 & 34 & 49 \\
\hline Calcul. Max. Thrust (N) & & 2.0 & & & 2.7 & \\
\hline Calculated Mass Decomposed (mg) & & 70 & & & 167 & \\
\hline
\end{tabular}

Table I: Summary of Test Results. 


\section{Future Directions}

The compound under evaluation, LAX 112 , thus far has not provided satisfactory results. Nonrepeatable ignition temperatures and incomplete decomposition reduce performance. Alternate gas generator compounds are being studied for evaluation. Among these candidates are alternate heterocyclic nitrogen compounds and cubanes. The most promising candidates will be selected for experimental evaluation.

The FT-IR spectra obtained from the compound suggest that significant absorption exists around 2.94. 3.04. 3.19. and 6.16 $\mu \mathrm{m}$ wavelength. Laser ignition testing at one of these wavelengths has the highest potential to accomplish laser ignition with the least required input power, a necessity for use on power limited micro-spacecraft. Future ignition tests will be directed to testing with such lasers. Alternative mechanisms, such as the use of pyrotechnic paste, to initiate the decomposition at wavelengths commonly used by diode lasers for communications purposes are under investigation.

MEMS components have been fabricated. Pellet sizes could not be fabricated to accommodate the $600 \mu \mathrm{m}$ MEMS thruster chamber height, so specially designed spacers are required between two half thrusters to accommodate the gas generator compound pellets. Future experiments are planned to tests this "split" thruster concept.

During future tests, temperature and thrust levels need to be measured in order to better validate the concept. These measurements are scheduled within a low pressure $(1 \mathrm{~Pa})$ facility. Thrust stands able to measure thrust levels and impulse bit sizes described in this paper are available. An obstacle is the powder material generated by incomplete decomposition. This powder could corrupt vacuum pumps and gauges required to accomplish the measurement. Improved decomposition should precede the thrust measurement.

\section{Summary}

A decomposing solid thruster concept, which creates a more benign thermal and chemical environment than solid propellant combustion, while maintaining performance similar to solid combustion, is described. A Micro-Electromechanical thruster concept with diode laser and fiber-optic ignition is proposed, and thruster components fabricated with MEMS technology are presented.

A solid gas generator compound with a good theoretical performance is evaluated. Tests to evaluate initiation of the decomposition are performed under low pressure, with a heated nichrome wire as ignition source. These tests show a repeatable ignition temperature between $188^{\circ} \mathrm{C}$ and $190{ }^{\circ} \mathrm{C}$, lower than the literature value of $220{ }^{\circ} \mathrm{C}$. Decomposition, however, is incomplete, possibly due to the low pressure.

FT-IR measurements of the original compound and residue material show partial decomposition of the residue, varying with distance between location where the powder is collected and the original mounting location.

Alternate tests are performed with compound material inside a miniature axisymmetric thruster, with decomposition initiated by heating the solid copper compound holder. Ignition temperature varies substantially, from $165{ }^{\circ} \mathrm{C}$ to $192{ }^{\circ} \mathrm{C}$. Decomposition is again incomplete, with the degree of decomposition varying significantly between tests. Chamber pressures up to $1.1 \mathrm{MPa}$ are obtained, with maximum thrust level about $2.8 \mathrm{~N}$

\section{References}

1. Bzibziak, R.: "Miniature Cold Gas Thrusters," AIAA 92-3256, Nashville, July 6-8, 1992.

2. Janson, S., Chemical and Electric Micropropulsion Concepts for Nanosatellites, Paper AIAA 94-2998, $30^{\text {th }}$ JPC Conference, Indianapolis, IN (1994).

3. Mueller, J., Thruster Options for Microspacecraft: A Review and Evaluation of Existing Hardware and Emerging Technologies, AIAA Paper 97-3058, $33^{\text {rd }}$ JPC Conf., Seattle, WA (1997). 


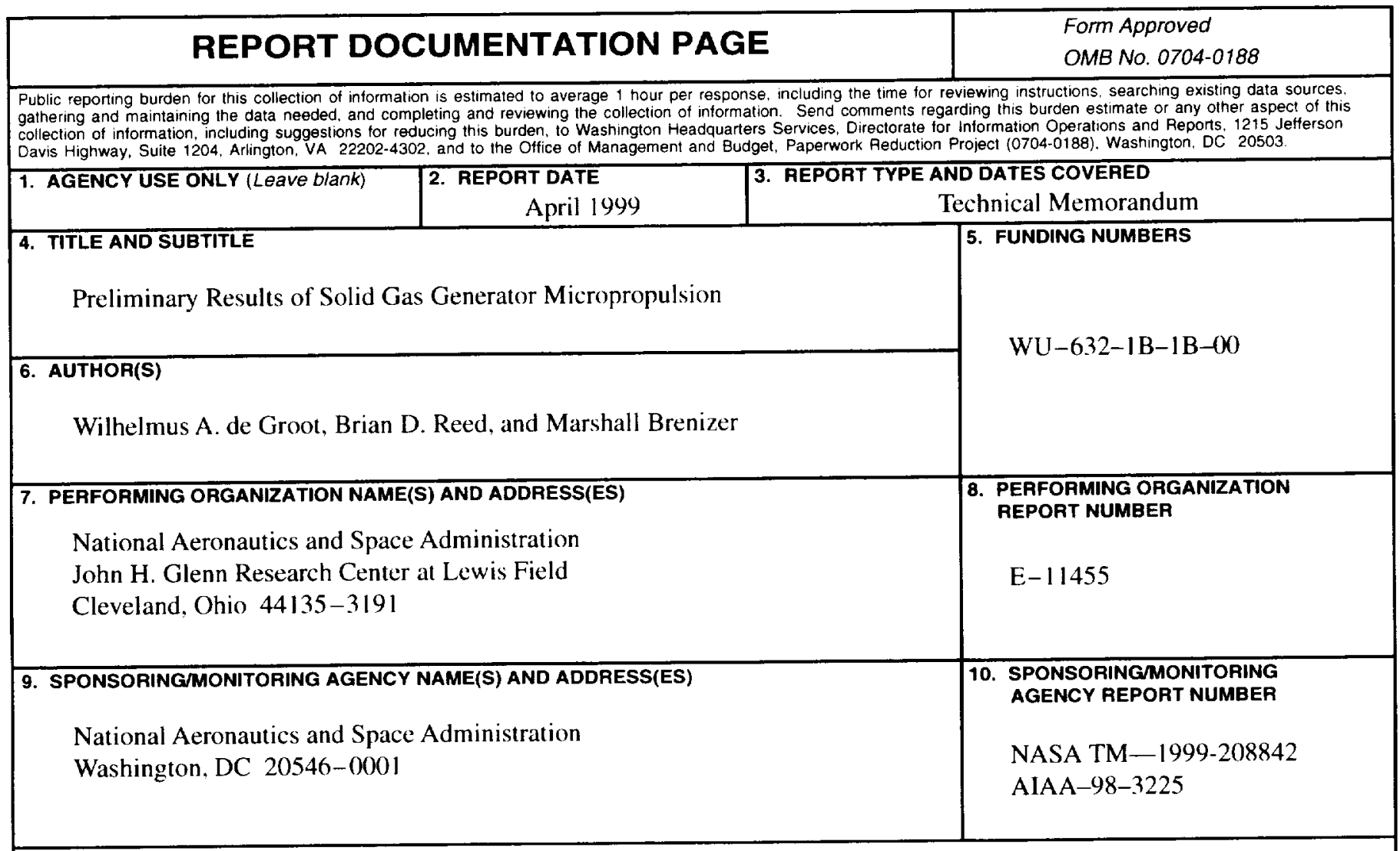

\section{SUPPLEMENTARY NOTES}

Prepared for the 34th Joint Propulsion Conference and Exhibit cosponsored by the AIAA, ASME, SAE, and ASEE, Cleveland. Ohio, July 13-15, 1998. Wilhelmus A. de Groot, Dynacs, Inc., 2001 Aerospace Parkway, Brook Park, Ohio 44142: Brian D. Reed, NASA Glenn Research Center; and Marshall Brenizer, Massachusetts Institute of Technology, Cambridge, Massachusetts 02139. Responsible person, Wilhelmus A. de Groot, organization code 5430, (216) 977-7485.

\begin{tabular}{l|l|l|l|} 
12a. DISTRIBUTION/AVAILABILITY STATEMENT & DISTRIBUTION CODE
\end{tabular}

Unclassified - Unlimited

Subject Category: 20
Distribution: Nonstandard

This publication is available from the NASA Center for AeroSpace Information, (301) 621-0390.

13. ABSTRACT (Maximum 200 words)

A decomposing solid thruster concept, which creates a more benign thermal and chemical environment than solid propellant combustion, while maintaining performance similar to solid combustion, is described. A Micro-Electro-Mechanical thruster concept with diode laser and fiber-optic initiation is proposed, and thruster components fabricated with MEMS technology are presented. A high nitrogen content solid gas generator compound is evaluated and tested in a conventional axisymmetric thrust chamber with nozzle throat area ratio of 100 . Results show incomplete decomposition of this compound in both low pressure $(1 \mathrm{kPa}$ ) and high pressure ( $1 \mathrm{MPa}$ ) environments, with decomposition of up to $80 \%$ of the original mass. Chamber pressures of 1.1 MPa were obtained, with maximum calculated thrust of approximately $2.7 \mathrm{~N}$. Resistively heated wires and resistively heated walls were used to initiate decomposition. Initiation tests using available lasers were unsuccessful, but infrared spectra of the compound show that the laser initiation tests used inappropriate wavelengths for optimal propellant absorption. Optimal wavelengths for laser ignition were identified. Data presented are from tests currently in progress. Alternative solid gas generator compounds are being evaluated for future tests.

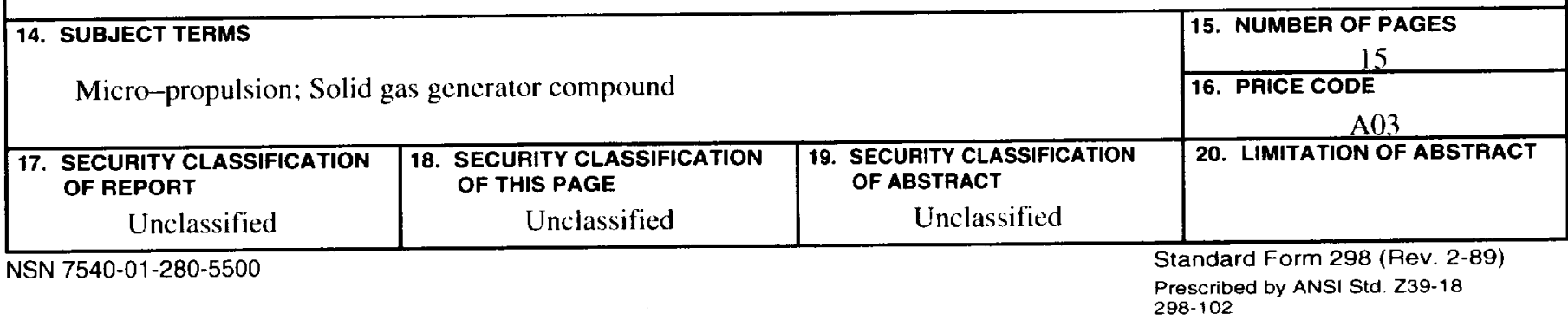


4. Epstein, A.,"Micro Heat Engines, Gas Turbines, and Rocket Engines-The MIT Microengine Project," AIAA-97-1773, 28 ${ }^{\text {th }}$ AIAA Fluid Dynamics Conf., Snowmass Village, CO, 1997

5. de Groot, W. A., and Oleson, S.R., "Chemical Microthruster Options," AIAA Paper 96-2868, $32^{\text {nd }}$ JPC Conf... Lake Buena Vista, FL. 1996.

6. Sutton, G. P. Rocket Propulsion Elements, Sixth Edition, John Wiley and Sons, New York, 1992.

7. Li, J., and Xu. Y.. "Some Recent Investigation in Solid Propellant Technology for Gas Generator," AIAA 90-2335, July 1990.
8. Maycock, J.N. and Pai Verneker, V.R. "A Photochemical Microrocket for Attitude Control," Journal of Spacecraft and Rockets, Vol.6, No.3, March 1969, pp.336-337.

9. Grisnik, S.P., Smith, T.A., and Saltz, L.E., "Experimental Study of Low Reynolds Number Nozzles," AIAA-87-0992, $19^{\text {th }}$ IEPC Conference, Colorado Springs, Co, 1987

10. Coburn, M.D., Hiskey, M.A., Lee, K.-Y., Ott, D.G., and Stinecipher, M.M.. "Oxidations of 3,6-Diamino-1,2,4,5tetrazine and 3,6-Bis(S,Sdimethylsulfilimino)-1,2,4,5-tetrazine," J. Heterocyclic Chem., 30, pp. 1593-1595, 1993. 